in semi-structured interviews, focus groups and electronic open end questionnaires.

Results In the first year, 201 received the service. 184 (92\%) had cancer, $36 \%$ aged over 80 years. 57 (28\%) lived alone. $181(90 \%)$ recipients died. 73\% (132) died at home (72\% (120) with cancer), 6\% (29) in the hospice, 12 (6.5\%) care home, $1(0.5 \%)$ in an ambulance on their way home, 7 (4\%) hospital. 51 patients lived alone, 69\% (35) died at home. Healthcare Professionals reported the impact of the different elements of the service (accompanied transfer home; multiprofessional (including doctors) crisis intervention team and a flexible sitting service) as being instrumental in helping patients to remain at home. The additional service supplements existing services, enables a speedier discharge home and supports carers to enable them to continue coping. It is of interest that in the second year a $73 \%$ home death rate has been maintained.

Conclusions This novel bespoke service provides different elements of a Hospice at Home service, a tailor made package to meet individual and local area needs. This service appears to be having a major impact on place of death and is enabling patients to die in their place of choice

\section{P95 'MADE TO FIT': FILLING CRITICAL GAPS WITH A HOSPICE AT HOME SERVICE}

Catherine Baldry, Barbara Jack, ${ }^{2}$ Karen Groves, ${ }^{1}$ Kathryn Gaunt, ${ }^{3}$ Janice Sephton, ${ }_{1}^{1}$ Alison Whelan' ${ }^{1}$ Queenscourt Hospice, Southport, UK; ${ }^{2} E d g e$ Hill University, Ormskirk, UK; ${ }^{3}$ Mersey Deanery, Liverpool, UK

10.1136/bmjspcare-2011-000105.95

Background Promoting the choice to die at home is central to UK policies and strategies. Nationally, various models of Hospice at Home service support this choice. Locally a service was developed to fill gaps in, and work together with, existing community services providing a bespoke Hospice at Home Service outreaching from the hospice hub.

Method Retrospective cohort study and stakeholder evaluation during a 1 year pilot study in the North West of England. Data (demographic and service intervention) was collected on 201 service recipients. 55 Healthcare Professionals; (General Practitioners, District Nurses, Community Specialist Palliative Care Nurses and Hospital Discharge Coordinator) participated 